\title{
On the acceleration of the convergence of singular operators in Gaussian basis sets
}

\author{
Krzysztof Pachucki ${ }^{\text {a) }}$ \\ Institute of Theoretical Physics, Warsaw University, Hoża 69, 00-681 Warsaw, Poland \\ Wojciech Cencek ${ }^{\text {b) }}$ \\ Department of Physics and Astronomy, University of Delaware, Newark, Delaware 19716 \\ Jacek Komasa ${ }^{\mathrm{c})}$ \\ Quantum Chemistry Group, Department of Chemistry, A. Mickiewicz University, Grunwaldzka 6, \\ 60-780 Poznań, Poland
}

(Received 28 January 2005; accepted 17 February 2005; published online 6 May 2005)

\begin{abstract}
Gaussian type wave functions do not reproduce the interparticle cusps which result in a slow convergence of the expectation values of the operators involved in calculations of the relativistic and QED energy corrections. Methods correcting this deficiency are the main topic discussed in this paper. Benchmark expectation values of the singular operators for several few-electron systems are presented. () 2005 American Institute of Physics. [DOI: 10.1063/1.1888572]
\end{abstract}

\section{INTRODUCTION}

The method of exponentially correlated Gaussian (ECG) wave functions provides a means of obtaining very accurate solutions of the Schrödinger equation for small, few-electron atomic and molecular systems. In the last decade it was shown that the method is capable of supplying the most accurate variational estimations of the nonrelativistic energy of two- to four-electron systems. ${ }^{1-7}$ Recently, the ECG wave functions also proved useful in determining relativistic and radiative corrections. ${ }^{1,4,8-11}$ A precise determination of the corrections requires access to high quality fully correlated wave functions. However, the expectation value of the energy is not the only factor determining the quality of the wave function. It is commonly known that the Gaussian-type wave functions, apart from their advantages, also have some drawbacks which lower their usefulness in certain applications. These drawbacks are related to the Kato cusp condition which is not fulfilled by the Gaussian functions. The exception are wave functions with a linear correlation factor like the LECG function. ${ }^{12,13}$ Such wave functions are also employed in the family of the R12 methods based on Kutzelnigg's idea. ${ }^{14,15}$

In this paper we shall concentrate on relativistic and QED energy corrections. The singular operators involved in these corrections are very demanding with respect to local properties of the wave function. In this case the Gaussiantype wave functions exhibit a convergence which can hardly be recognized as satisfactory. The operators in mind are the one- and two-electron Dirac delta operators, $\delta(\boldsymbol{r})$, the relativistic kinetic energy operator $\nabla^{4}$, and the distributional operator $P\left(1 / r_{i j}^{3}\right)$ entering the QED energy correction. In this paper we describe two, in principle general, regularization

\footnotetext{
${ }^{a}$ Electronic mail: krp@fuw.edu.pl

b)Electronic mail: cencek@ physics.udel.edu

${ }^{\mathrm{c})}$ Electronic mail: komasa@man.poznan.pl
}

methods which allow the convergence of the pertinent expectation values to be significantly improved. We propose to use the name Drachmanization for the first methodology as it was introduced by R. J. Drachman. ${ }^{16}$ The other method, which is introduced here, is based on a suitable integral transform and an asymptotic formula for the resulting integrand. Referring to these methods we will use the abbreviations Dr and IT for Drachmanization and integral transform, respectively. We illustrate our approaches by one molecular $\left(\mathrm{H}_{2}\right)$ and three atomic $(\mathrm{He}, \mathrm{Li}$, and $\mathrm{Be})$ cases using accurate ECG wave functions. The atomic units are used throughout the paper.

\section{THE WAVE FUNCTION}

The energies and wave functions of the studied systems were obtained by variationally solving the stationary Schrödinger equation $\hat{H} \Psi=E \Psi$ with the nonrelativistic clamped nuclei Hamiltonian

$$
\begin{aligned}
\hat{H}= & -\frac{1}{2} \sum_{i=1}^{n} \nabla_{i}^{2}+\sum_{i=1}^{n-1} \sum_{j=i+1}^{n} \frac{1}{\left|\boldsymbol{r}_{i}-\boldsymbol{r}_{j}\right|}-\sum_{I=1}^{N} \sum_{i=1}^{n} \frac{Z_{I}}{\left|\boldsymbol{r}_{i}-\boldsymbol{R}_{I}\right|} \\
& +\sum_{I=1}^{N-1} \sum_{J=I+1}^{N} \frac{Z_{I} Z_{J}}{\left|\boldsymbol{R}_{I}-\boldsymbol{R}_{J}\right|},
\end{aligned}
$$

where $\boldsymbol{r}_{i}$ and $\boldsymbol{R}_{I}$ are, respectively, the electronic and nuclear coordinates, and $Z_{I}$ are the nuclear charges. Our trial wave function was assumed in the form of a $K$-term linear expansion in an $n$-electron basis

$$
\Psi(\boldsymbol{r}, \boldsymbol{\sigma})=\hat{\mathcal{A}}\left(\Xi_{n, S, M_{S}}(\boldsymbol{\sigma}) \hat{\mathcal{P}} \sum_{k=1}^{K} c_{k} \psi_{k}(\boldsymbol{r})\right),
$$

where $\hat{\mathcal{A}}$ and $\hat{\mathcal{P}}$ are the permutation and spatial symmetry projectors, respectively, and $\Xi_{n, S, M_{S}}(\boldsymbol{\sigma})$ is an $n$-electron spin function with the spin quantum numbers $S$ and $M_{S}$. As the $n$-electron spatial basis functions, $\psi_{k}$, we employed the ex- 
ponentially correlated Gaussian (ECG) functions of Singer, ${ }^{17}$

$$
\psi_{k}(\boldsymbol{r})=\exp \left[-\left(\boldsymbol{r}-\boldsymbol{s}_{k}\right) \boldsymbol{A}_{k}\left(\mathbf{r}-\boldsymbol{s}_{k}\right)^{T}\right],
$$

where the superscript $T$ denotes a vector transpose. The linear parameters, $c_{k}$, were determined by the inverse iteration method of solving the standard general symmetric eigenvalue problem. The nonlinear parameters, contained in the vectors $\boldsymbol{s}_{k}$ and the positive definite matrices $\boldsymbol{A}_{k}$, were found in an extensive variational optimization process. Detailed description of the ECG method including the optimization strategy employed may be found in Refs. 5 and 18-21.

\section{REGULARIZATION METHODS}

\section{A. Drachmanization (Dr)}

The first regularization method is based on the idea described in 1981 by Drachman, ${ }^{16}$ who expressed the expectation value of $\delta\left(\boldsymbol{r}_{j k}\right)$ in an equivalent form containing global operators with the exact wave function $\Psi$ and its energy $E_{\Psi}$, namely

$$
\begin{aligned}
4 \pi\left\langle\Psi\left|\delta\left(\boldsymbol{r}_{j k}\right)\right| \Psi\right\rangle= & 2\left\langle\Psi\left|\frac{1}{r_{j k}}\left(E_{\Psi}-\hat{V}\right)\right| \Psi\right\rangle \\
& -\sum_{i}\left\langle\nabla_{i} \Psi\left|\frac{1}{r_{j k}}\right| \nabla_{i} \Psi\right\rangle,
\end{aligned}
$$

where $\hat{V}$ is the potential energy operator. Analogously we can reduce the singularity of the electron-nucleus Dirac delta operator,

$$
\begin{aligned}
4 \pi\left\langle\Psi\left|\delta\left(\boldsymbol{r}_{k I}\right)\right| \Psi\right\rangle= & 4\left\langle\Psi\left|\frac{1}{r_{k I}}\left(E_{\Psi}-\hat{V}\right)\right| \Psi\right\rangle \\
& -2 \sum_{i}\left\langle\nabla_{i} \Psi\left|\frac{1}{r_{k I}}\right| \nabla_{i} \Psi\right\rangle .
\end{aligned}
$$

In a similar way, the relativistic kinetic energy term can be evaluated using

$$
\left\langle\Psi\left|\sum_{i} \nabla_{i}^{4}\right| \Psi\right\rangle=4\left\langle\Psi\left|\left(E_{\Psi}-\hat{V}\right)^{2}\right| \Psi\right\rangle-2 \sum_{i>j}\left\langle\nabla_{i}^{2} \Psi \mid \nabla_{j}^{2} \Psi\right\rangle
$$

The QED energy correction contains a distribution $P$ which is defined as the limit

$$
\begin{aligned}
\left\langle\phi\left|P\left(\frac{1}{r^{3}}\right)\right| \psi\right\rangle \equiv & \lim _{a \rightarrow 0} \int \phi^{*}(\boldsymbol{r}) \psi(\boldsymbol{r})\left[\frac{1}{r^{3}} \Theta(r-a)\right. \\
& +4 \pi \delta(\boldsymbol{r})(\gamma+\ln a)] d \boldsymbol{r}
\end{aligned}
$$

with $\Theta$ and $\gamma$ being the step function and the Euler constant, respectively. In order to obtain a more regular expression for $P$ we need to find a function for which $\nabla^{2}$ gives $P\left(1 / r_{j k}^{3}\right)$, namely

$$
\nabla_{j}^{2}\left(\frac{1+\gamma+\ln r_{j k}}{r_{j k}}\right)=-P\left(\frac{1}{r_{j k}^{3}}\right) .
$$

Next, we perform similar rearrangements as before and obtain

$$
\begin{aligned}
\left\langle\Psi\left|P\left(\frac{1}{r_{j k}^{3}}\right)\right| \Psi\right\rangle= & \sum_{i}\left\langle\nabla_{i} \Psi\left|\frac{\ln r_{j k}}{r_{j k}}\right| \nabla_{i} \Psi\right\rangle \\
& +\langle\Psi| 4 \pi(1+\gamma) \delta\left(\boldsymbol{r}_{j k}\right) \\
& +2\left(E_{\Psi}-\hat{V}\right) \frac{\ln r_{j k}}{r_{j k}}|\Psi\rangle .
\end{aligned}
$$

As can be seen, except for $\delta\left(\boldsymbol{r}_{j k}\right)$, which can be handled according to Eq. (4), singular operators are no longer present on the right-hand side of Eq. (9), but instead new types of integrals appear. In the ECG basis these integrals can be evaluated by means of elementary and the Clausen $\mathrm{Cl}_{2}$ (Ref. 22) functions, for example

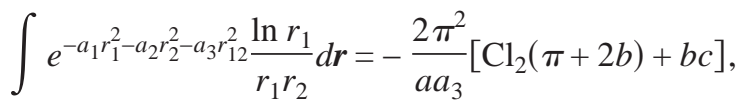

where

$$
\begin{aligned}
& a=\sqrt{a_{1} a_{2}+a_{1} a_{3}+a_{2} a_{3}}, \\
& b=\arctan \left(\frac{a_{3}}{a}\right), \\
& c=\gamma+\ln \left(\frac{4 a^{2}}{a_{2}+a_{3}}\right) .
\end{aligned}
$$

The above-described methodology is an extension of the original Drachman's idea to a couple of new operators, therefore, we dare to call this approach Drachmanization. Formulas (4)-(6) and (9) are general but an analytic evaluation of the right-hand-side integrals in the ECG basis is possible only for atoms. This prompted us to search for another method which, while preserving the accuracy, would be equally effective for atoms and molecules.

\section{B. Integral transform (IT)}

The idea of the IT approach is to move the singularity out of an expectation value to the new integral, and to derive the correct asymptotic formula for the integrand from the analytic behavior of the exact solution of the Schrödinger equation. This is the simplest approach, which can be applied to atoms as well as to molecules.

\section{1. $\langle\Psi|\delta(r)| \Psi\rangle$}

For the Dirac delta operators this method works as follows. One uses the Poisson equation

$$
\nabla^{2}\left(\frac{1}{r}\right)=-4 \pi \delta(\boldsymbol{r})
$$

and replaces $1 / r$ by an integral with the Gaussian function

$$
\frac{1}{r}=\frac{2}{\sqrt{\pi}} \int_{0}^{\infty} e^{-r^{2} t^{2}} d t
$$

This combination leads to the following integral form of the Dirac delta function: 


$$
\langle\Psi|\delta(\boldsymbol{r})| \Psi\rangle=\frac{1}{2 \pi^{3 / 2}} \int_{0}^{\infty}\left\langle\Psi\left|2 t^{2}\left(3-2 t^{2} r^{2}\right) e^{-t^{2} r^{2}}\right| \Psi\right\rangle d t .
$$

In a well-optimized ECG basis set the matrix element of $e^{-t^{2} r^{2}}$ is highly accurate, provided that $t$ is not very large, i.e., $t<t_{\Lambda}$. Therefore, the integral over $t>t_{\Lambda}$ is evaluated using an analytically derived asymptotic formula. This formula results from the Kato cusp condition, which is fulfilled by the exact solution of the Schrödinger equation with Coulomb interactions. If one denotes by $f(t)$ the integrand in Eq. (14), then for large $t$ we use the expansion

$$
f(t)=\frac{4 Z}{\sqrt{\pi} t^{2}}\langle\Psi|\delta(\boldsymbol{r})| \Psi\rangle\left(1+\frac{A_{1}}{t}+\frac{A_{2}}{t^{2}}+\frac{A_{3}}{t^{3}}+\cdots\right),
$$

where $A_{i}$ depend on the state under consideration, $\Psi$, and are obtained by fitting to a large grid of points of $f(t)$. For the electron-nucleus Dirac delta, $Z$ is the nuclear charge whereas for the interelectron delta, $Z=-\frac{1}{2}$. It remains to make a proper choice of the value for $t_{\Lambda}$, which depends on the numerical accuracy of $f(t)$. This accuracy is estimated by calculating $f(t)$ for several lengths of basis sets and we found that most precise results with our wave functions are obtained with $t_{\Lambda} \approx 100$. In performing the integration in Eq. (14) one, at first, neglects the asymptotic formula, obtains approximate value for the Dirac delta, and in the next step uses it for the calculation of the integral over the asymptotic region. The result converges quickly with only a few iterations.

\section{2. $\left\langle\Psi\left|P\left(1 / r^{3}\right)\right| \Psi\right\rangle$}

A similar approach can be applied to the calculation of the Araki-Sucher term, which is defined in Eq. (7). One transforms this term with the help of

$$
-\frac{\ln r+\gamma}{r^{2}}=\int_{0}^{\infty} t(2 \ln t-\gamma) e^{-t^{2} r^{2}} d t
$$

and of the integration by parts to the following form:

$$
\begin{aligned}
\int P\left(\frac{1}{r_{i j}^{3}}\right) \Psi^{2}(\boldsymbol{r}) d \boldsymbol{r} & =-\int \frac{\ln r_{i j}+\gamma}{r_{i j}^{2}} \frac{\partial}{\partial r_{i j}} \Psi^{2}(\boldsymbol{r}) d \boldsymbol{r} \\
& =\int_{0}^{\infty}(2 \ln t-\gamma) f(t) d t,
\end{aligned}
$$

where

$$
f(t)=\int 2 t\left(t^{2} r_{i j}-\frac{1}{r_{i j}}\right) e^{-t^{2} r_{i j}^{2}} \Psi^{2}(\boldsymbol{r}) d \boldsymbol{r} .
$$

As in the case of the Dirac delta function, the integral with $t<t_{\Lambda}$ is calculated directly with $f(t)$, while for $t>t_{\Lambda}$ one applies the asymptotic expansion

$$
f(t)=\frac{\pi^{3 / 2}}{2 t^{2}}\left\langle\Psi\left|\delta\left(\boldsymbol{r}_{i j}\right)\right| \Psi\right\rangle\left(1+\frac{A_{1}}{t}+\frac{A_{2}}{t^{2}}+\frac{A_{3}}{t^{3}}+\cdots\right)
$$

and calculates $A_{i}$ by fitting to $f(t)$. The value of $t_{\Lambda}$ is chosen as equal to that in the corresponding calculation of the Dirac delta.
TABLE I. The nonrelativistic energy, $E$, its relative error, $\Delta E$, and the largest expansion length, $K$, of the ECG wave function applied.

\begin{tabular}{ccccc}
\hline \hline System & $K$ & $E / E_{H}$ & $\Delta E / E_{H}$ & Reference \\
\hline $\mathrm{He}$ & 600 & -2.903724377022 & $4.1 \times 10^{-12}$ & 1 \\
$\mathrm{Li}$ & 2000 & -7.478060315 & $1.2 \times 10^{-9}$ & 10 \\
$\mathrm{Be}$ & 1600 & -14.667355536 & $3.1 \times 10^{-8}$ & 31 \\
$\mathrm{H}_{2}$ & 1200 & -1.174475714037 & $1.6 \times 10^{-10}$ & 1 \\
\hline \hline
\end{tabular}

\section{3. $\left\langle\Psi\left|\nabla^{4}\right| \Psi\right\rangle$}

A different approach is applied in the case of $\left\langle\Psi\left|\nabla_{1}^{4}\right| \Psi\right\rangle=\left\langle\Psi\left|p_{1}^{4}\right| \Psi\right\rangle$. We switch to the momentum representation and calculate the one-electron momentum density function, $I\left(p_{1}\right)$, employing the Fourier transform of the coordinate wave function

$$
\widetilde{\Psi}(\boldsymbol{p}, \boldsymbol{\sigma})=\int \Psi(\boldsymbol{r}, \boldsymbol{\sigma}) e^{-i \boldsymbol{p r}} d \boldsymbol{r} .
$$

We note in passing that the Fourier transform of the ECG basis function of Eq. (3),

$$
\widetilde{\psi}_{k}(\boldsymbol{p})=\left|\widetilde{\boldsymbol{A}}_{k}\right|^{-3 / 2} \exp \left[-\left(\boldsymbol{p}-\widetilde{\boldsymbol{s}}_{k}\right) \widetilde{\boldsymbol{A}}_{k}\left(\boldsymbol{p}-\widetilde{\boldsymbol{s}}_{k}\right)^{T}+\widetilde{\boldsymbol{C}}\right]
$$

with $\widetilde{\boldsymbol{s}}_{k}=-2 i \mathbf{s}_{k} \boldsymbol{A}_{k}, \widetilde{\boldsymbol{A}}_{k}=\frac{1}{4} \boldsymbol{A}_{k}^{-1}$, and $\widetilde{C}=-\boldsymbol{s}_{k} \boldsymbol{A}_{k} \boldsymbol{s}_{k}^{T}$, preserves the shape and all the advantages of the original ECG function, which enables the evaluation of integrals in the momentum space to be readily accomplished. ${ }^{7,23}$ The integration with respect to all the momenta but $p_{1}$ leads to

$$
\left\langle\Psi\left|\sum_{i} p_{i}^{4}\right| \Psi\right\rangle=n \int p_{1}^{4} \widetilde{\Psi}(\boldsymbol{p}) \widetilde{\Psi}^{*}(\boldsymbol{p}) d \boldsymbol{p}=\int_{0}^{\infty} p_{1}^{4} I\left(p_{1}\right) d p_{1},
$$

where

$$
I(p)=4 \pi n p^{2} \int \widetilde{\Psi}\left(\boldsymbol{p}, \boldsymbol{p}_{2}, \ldots, \boldsymbol{p}_{n}\right) \widetilde{\Psi}^{*}\left(\boldsymbol{p}, \boldsymbol{p}_{2}, \ldots, \boldsymbol{p}_{n}\right) \prod_{i=2}^{n} d \boldsymbol{p}_{i} .
$$

The density function $I(p)$, as obtained from Gaussian geminals, is accurate for not too large values of the momentum. Therefore, the last integral in Eq. (22) is evaluated directly for $p<p_{\Lambda}$ whereas for $p>p_{\Lambda}$ we use a large- $p$ asymptotic expansion. This expansion can be obtained as follows. The large momentum behavior is related to small $r$ in the coordinate representation. For example, for hydrogenic states

$$
I(p)=\frac{32}{p^{6}} Z^{2}\langle\Psi|\delta(\boldsymbol{r})| \Psi\rangle+O\left(p^{-8}\right) .
$$

The generalization to arbitrary (but nonrelativistic) atoms with $n$ electrons is

$$
\begin{aligned}
I(p)= & \frac{32}{p^{6}} \sum_{i=1}^{n}\left(Z^{2}\left\langle\Psi\left|\delta\left(\boldsymbol{r}_{i}\right)\right| \Psi\right\rangle+\frac{1}{2} \sum_{j>i}^{n}\left\langle\Psi\left|\delta\left(\boldsymbol{r}_{i j}\right)\right| \Psi\right\rangle\right) \\
& +O\left(p^{-8}\right),
\end{aligned}
$$

which will be proven in the following. The result for molecules is more complicated and involves an oscillatory term 
TABLE II. Helium atom. Convergence of the expectation values computed directly, using the Drachmanization (Dr), and by the integral transform (IT). An implicit summation over all the electrons and electron pairs is assumed in the notation used here and in Tables III-VI.

\begin{tabular}{|c|c|c|c|}
\hline$K$ & Direct & Dr & IT \\
\hline \multicolumn{4}{|c|}{$\left\langle\delta\left(\boldsymbol{r}_{i}\right)\right\rangle$} \\
\hline 75 & 3.60821679 & 3.62080582 & 3.62061000 \\
\hline 150 & 3.61778237 & 3.62085606 & 3.62085450 \\
\hline 300 & 3.61959530 & 3.62085821 & 3.62085839 \\
\hline 600 & 3.62061121 & 3.62085862 & 3.62085863 \\
\hline "Exact"a & \multicolumn{3}{|c|}{$3.6208586377(3)$} \\
\hline \multicolumn{4}{|c|}{$\left\langle\delta\left(\boldsymbol{r}_{i j}\right)\right\rangle$} \\
\hline 75 & 0.106951650 & 0.106341763 & 0.106414468 \\
\hline 150 & 0.106465966 & 0.106345233 & 0.106346293 \\
\hline 300 & 0.106378464 & 0.106345356 & 0.106345437 \\
\hline 600 & 0.106358176 & 0.106345369 & 0.106345383 \\
\hline "Exact"b & & $0.106345370636(2)$ & \\
\hline \multicolumn{4}{|c|}{$\left\langle p_{i}^{4}\right\rangle$} \\
\hline 75 & 107.84986 & 108.18161 & 108.173524 \\
\hline 150 & 108.09726 & 108.17755 & 108.176048 \\
\hline 300 & 108.14396 & 108.17653 & 108.176115 \\
\hline 600 & 108.16977 & 108.17629 & 108.176135 \\
\hline "Exact"a & & $108.1761344(8)$ & \\
\hline \multicolumn{4}{|c|}{$\left\langle P\left(1 / r_{i j}^{3}\right)\right\rangle$} \\
\hline 75 & 0.9484220 & 0.9894856 & 0.9782955 \\
\hline 150 & 0.9787062 & 0.9892845 & 0.9891200 \\
\hline 300 & 0.9858764 & 0.9892748 & 0.9892618 \\
\hline 600 & 0.9878049 & 0.9892735 & 0.9892721 \\
\hline "Exact"c & & $0.9892724(13)$ & \\
\hline
\end{tabular}

${ }^{\mathrm{a}}$ Reference 24.

${ }^{\mathrm{b}}$ Reference 25.

${ }^{\mathrm{c}}$ Reference 26.

of the order of $p^{-7}$. Let us present more detailed derivation for the diatomic molecule $A B$ consisting of two electrons. The generalization to arbitrary molecule will be obvious. The wave function is $\Psi=\Psi\left(\boldsymbol{r}_{1}, \boldsymbol{r}_{2}\right)$. We perform the Fourier transform in the variable $\boldsymbol{r}_{1}$, while keeping $\boldsymbol{r}_{2}$ intact,

$$
\widetilde{\Psi}\left(\boldsymbol{p}_{1}, \boldsymbol{r}_{2}\right)=\int \Psi\left(\boldsymbol{r}_{1}, \boldsymbol{r}_{2}\right) e^{-i p_{1} \boldsymbol{r}_{1}} d \boldsymbol{r}_{1}
$$

The large $\mathrm{p}_{1}$ asymptotics is related to the cusps at both nuclei and at the other electron

$$
\begin{aligned}
\widetilde{\Psi}\left(\boldsymbol{p}_{1}, \boldsymbol{r}_{2}\right) \approx & \frac{8 \pi Z_{A}}{p_{1}^{4}} \Psi\left(\boldsymbol{R}_{A}, \boldsymbol{r}_{2}\right) e^{-i p_{1} \boldsymbol{R}_{A}} \\
& +\frac{8 \pi Z_{B}}{p_{1}^{4}} \Psi\left(\boldsymbol{R}_{B}, \boldsymbol{r}_{2}\right) e^{-i p_{1} \boldsymbol{R}_{B}} \\
& -\frac{1}{2} \frac{8 \pi}{p_{1}^{4}} \Psi\left(\boldsymbol{r}_{2}, \boldsymbol{r}_{2}\right) e^{-i p_{1} \boldsymbol{r}_{2}}
\end{aligned}
$$

TABLE III. Lithium atom. Expectation values computed from the 2000-term ECG wave function by means of three methods: directly, using the Drachmanization (Dr), and by the integral transform (IT) in comparison with accurate Yan and Drake values (Ref. 27).

\begin{tabular}{lllll}
\hline \hline & $\left\langle\delta\left(\boldsymbol{r}_{j}\right)\right\rangle$ & $\left\langle\delta\left(\boldsymbol{r}_{i j}\right)\right\rangle$ & $\left\langle p_{i}^{4}\right\rangle$ & $\left\langle P\left(1 / r_{i j}^{3}\right)\right\rangle$ \\
\hline Direct & 13.8350 & 0.544669 & 628.157 & 0.2410 \\
Dr & 13.84260623 & 0.5443244 & 628.4531 & 0.27354 \\
IT & 13.84260963 & 0.5443266 & 628.4490 & 0.27337 \\
a & $13.842609642(55)$ & $0.54432979(31)$ & $628.449144(5)$ & $0.27367(26)$ \\
\hline
\end{tabular}

${ }^{\mathrm{a}}$ Reference 27. 
is then $(n=2)$

$$
\begin{aligned}
I(\boldsymbol{p})= & n \frac{4 \pi p^{2}}{(2 \pi)^{3}} \int \widetilde{\Psi}^{*}\left(\boldsymbol{p}, \boldsymbol{r}_{2}\right) \widetilde{\Psi}\left(\boldsymbol{p}, \boldsymbol{r}_{2}\right) d \boldsymbol{r}_{2} \\
\approx & n \frac{32}{p^{6}} \int\left[Z_{A} \Psi\left(\boldsymbol{R}_{A}, \boldsymbol{r}_{2}\right) e^{-i p \boldsymbol{R}_{A}}+Z_{B} \Psi\left(\boldsymbol{R}_{B}, \boldsymbol{r}_{2}\right) e^{-i p \boldsymbol{R}_{B}}\right. \\
& \left.-\frac{1}{2} \Psi\left(\boldsymbol{r}_{2}, \boldsymbol{r}_{2}\right) e^{-i p \boldsymbol{r}_{2}}\right]^{*} \times\left[Z_{A} \Psi\left(\boldsymbol{R}_{A}, \boldsymbol{r}_{2}\right) e^{-i \boldsymbol{p} \boldsymbol{R}_{A}}\right. \\
& \left.+Z_{B} \Psi\left(\boldsymbol{R}_{B}, \boldsymbol{r}_{2}\right) e^{-i p \boldsymbol{R}_{B}}-\frac{1}{2} \Psi\left(\boldsymbol{r}_{2}, \boldsymbol{r}_{2}\right) e^{-i p \boldsymbol{r}_{2}}\right] d \boldsymbol{r}_{2} .
\end{aligned}
$$

After expanding the product of two square brackets, the mixing terms with $e^{-i p R_{X}} e^{-i p r_{2}}$ do not contribute to the large $p$ asymptotics because of the integration over $\boldsymbol{r}_{2}$,

$$
\begin{aligned}
I(\boldsymbol{p})= & n \frac{32}{p^{6}} \int\left[Z_{A}^{2} \Psi^{2}\left(\boldsymbol{R}_{A}, \boldsymbol{r}_{2}\right)+Z_{B}^{2} \Psi^{2}\left(\boldsymbol{R}_{B}, \boldsymbol{r}_{2}\right)\right. \\
& +\frac{1}{4} \Psi^{2}\left(\boldsymbol{r}_{2}, \boldsymbol{r}_{2}\right)+2 Z_{A} Z_{B} \cos \left(\boldsymbol{p}\left(\boldsymbol{R}_{A}-\boldsymbol{R}_{B}\right)\right) \\
& \left.\times \Psi\left(\boldsymbol{R}_{A}, \boldsymbol{r}_{2}\right) \Psi\left(\boldsymbol{R}_{B}, \boldsymbol{r}_{2}\right)\right] d \boldsymbol{r}_{2}+O\left(p^{-8}\right) .
\end{aligned}
$$

One averages with respect to all orientations of $\boldsymbol{p}$ relative to $\boldsymbol{R}_{A}-\boldsymbol{R}_{B}$ and obtains

$$
\begin{aligned}
I(p)= & n \frac{32}{p^{6}}\left[Z_{A}^{2}\left\langle\Psi\left|\delta\left(\boldsymbol{r}_{1}-\boldsymbol{R}_{A}\right)\right| \Psi\right\rangle+Z_{B}^{2}\left\langle\Psi\left|\delta\left(\boldsymbol{r}_{1}-\boldsymbol{R}_{B}\right)\right| \Psi\right\rangle\right. \\
& +\frac{1}{4}\left\langle\Psi\left|\delta\left(\boldsymbol{r}_{1}-\boldsymbol{r}_{2}\right)\right| \Psi\right\rangle+Z_{A} Z_{B} \frac{2 \sin \left(p\left|\boldsymbol{R}_{A}-\boldsymbol{R}_{B}\right|\right)}{p\left|\boldsymbol{R}_{A}-\boldsymbol{R}_{B}\right|} \\
& \left.\times \int \Psi^{*}\left(\boldsymbol{R}_{A}, \boldsymbol{r}_{2}\right) \Psi\left(\boldsymbol{R}_{B}, \boldsymbol{r}_{2}\right) d \boldsymbol{r}_{2}\right]+O\left(p^{-8}\right) .
\end{aligned}
$$

\begin{tabular}{|c|c|c|c|}
\hline K & Direct & Dr & IT \\
\hline \multicolumn{4}{|c|}{$\left\langle\delta\left(\boldsymbol{r}_{i}\right)\right\rangle$} \\
\hline 100 & 33.379145 & 35.174770 & 35.324216 \\
\hline 200 & 34.897648 & 35.339657 & 35.362596 \\
\hline 400 & 35.029311 & 35.340791 & 35.365872 \\
\hline 800 & 35.297307 & 35.368099 & 35.368676 \\
\hline 1600 & 35.317352 & 35.368900 & 35.368880 \\
\hline$\infty$ & & \multicolumn{2}{|c|}{$35.3695(5)$} \\
\hline \multicolumn{4}{|c|}{$\left\langle\delta\left(\boldsymbol{r}_{i j}\right)\right\rangle$} \\
\hline 100 & 1.627741 & 1.604558 & 1.620185 \\
\hline 200 & 1.618229 & 1.605055 & 1.610551 \\
\hline 400 & 1.610911 & 1.605253 & 1.606134 \\
\hline 800 & 1.608106 & 1.605293 & 1.605446 \\
\hline 1600 & 1.606742 & 1.605303 & 1.605341 \\
\hline$\infty$ & & \multicolumn{2}{|c|}{$1.60532(1)$} \\
\hline \multicolumn{4}{|c|}{$\left\langle p_{i}^{4}\right\rangle$} \\
\hline 100 & 2137.407 & 2164.851 & 2164.780 \\
\hline 200 & 2153.618 & 2165.594 & 2165.503 \\
\hline 400 & 2155.764 & 2165.567 & 2165.681 \\
\hline 800 & 2161.966 & 2165.644 & 2165.613 \\
\hline 1600 & 2162.989 & 2165.637 & 2165.622 \\
\hline$\infty$ & & \multicolumn{2}{|c|}{$2165.64(1)$} \\
\hline \multicolumn{4}{|c|}{$\left\langle P\left(1 / r_{i j}^{3}\right)\right\rangle$} \\
\hline 100 & -8.55239 & -7.30429 & -7.55400 \\
\hline 200 & -8.12310 & -7.31837 & -7.40833 \\
\hline 400 & -7.73023 & -7.32454 & -7.34632 \\
\hline 800 & -7.55288 & -7.32622 & -7.33315 \\
\hline 1600 & -7.45534 & -7.32663 & -7.32856 \\
\hline$\infty$ & & \multicolumn{2}{|c|}{$-7.3268(3)$} \\
\hline
\end{tabular}

For arbitrary diatomic molecules $A B$ of the $\Sigma$ symmetry, the large momentum behavior of the angularly averaged $I(p)$ is

$$
\begin{aligned}
I(p)= & \frac{32}{p^{6}} \sum_{i=1}^{n}\left(Z_{A}^{2}\left\langle\Psi\left|\delta\left(\boldsymbol{r}_{i}-\boldsymbol{R}_{A}\right)\right| \Psi\right\rangle+Z_{B}^{2}\left\langle\Psi\left|\delta\left(\boldsymbol{r}_{i}-\boldsymbol{R}_{B}\right)\right| \Psi\right\rangle\right. \\
& \left.+\frac{1}{2} \sum_{j>i}^{n}\left\langle\Psi\left|\delta\left(\boldsymbol{r}_{i j}\right)\right| \Psi\right\rangle\right) \\
& +\frac{32}{p^{6}} Z_{A} Z_{B} \frac{2 \sin \left(p\left|\boldsymbol{R}_{A}-\boldsymbol{R}_{B}\right|\right)}{p\left|\boldsymbol{R}_{A}-\boldsymbol{R}_{B}\right|} \sum_{i=1}^{n} \rho_{i}\left(\left|\boldsymbol{R}_{A}-\boldsymbol{R}_{B}\right|\right) \\
& +O\left(p^{-8}\right),
\end{aligned}
$$

where

TABLE IV. Beryllium atom. Convergence of the expectation values computed directly, using the Drachmanization (Dr), and by the integral transform (IT). 
$\rho_{1}\left(\left|\boldsymbol{R}_{A}-\boldsymbol{R}_{B}\right|\right)=\int \Psi^{*}\left(\boldsymbol{R}_{A}, \boldsymbol{r}_{2}, \ldots, \boldsymbol{r}_{n}\right) \Psi\left(\boldsymbol{R}_{B}, \boldsymbol{r}_{2}, \ldots, \boldsymbol{r}_{n}\right) \prod_{i=2}^{n} d \boldsymbol{r}_{i}$

The higher order terms in the expansion (31) are obtained by fitting to $I(p)$ and the whole expansion is integrated analytically for $p>p_{\Lambda}$. The parameter $p_{\Lambda}$ is selected differently for each system as it depends on the quality of the wave function but, in a wide range of its choice, has no influence on the final result.

\section{RESULTS AND DISCUSSION}

The above-described methodology was applied to three atomic $(\mathrm{He}, \mathrm{Li}, \mathrm{Be})$ and one molecular $\left(\mathrm{H}_{2}\right)$ system. For their ground states accurate ECG wave functions are available from our previous papers (see Table I for details). Table I lists energy and its estimated accuracy corresponding to the largest expansion used for each out of the four systems.

The regularization methods were tested first on the helium atom case (Table II). For this system very accurate reference results, obtained from specialized exponential wave functions, are available. ${ }^{24-26}$ The direct and Dr values of $\left\langle\delta\left(\boldsymbol{r}_{i j}\right)\right\rangle$ were already published in Ref. 9, but are repeated here for the sake of completeness. Let us first note that the Dirac delta expectation value obtained in the direct ECG computation converges rather slowly, so that from the 600term expansion merely four significant figures of the exact value can be recovered despite the fact that the same wave function gives eleven figures of the exact energy. Both types of regularization allow us to get another four significant digits. A similar observation can be made for $\left\langle p_{i}^{4}\right\rangle$ but this time the IT approach is more effective and yields eight significant figures of the exact value. The expectation value of $P\left(1 / r_{i j}^{3}\right)$ is particularly slowly convergent and in direct computations only two significant digits are obtained. The convergence is dramatically improved after the application of any of the two regularization methods so that an accuracy of six decimals is reached.

In Table III the expectation values for the lithium atom computed directly from the 2000-term ECG wave function and by means of the regularization methods are confronted with the very accurate results obtained by Yan and Drake from an exponential wave function. ${ }^{27}$ In this case the direct computations give only three significant figures for the Dirac deltas and $\left\langle p_{i}^{4}\right\rangle$ and just the leading figure for $\left\langle P\left(1 / r_{i j}^{3}\right)\right\rangle$. We gain several more decimal digits from the regularized calculations. In the case of lithium the IT approach seems to be consistently superior to the Drachmanization, with the exception of the latter operator, where both techniques seem to perform approximately equally well but the precise comparison is impossible due to the uncertainty of the reference value.

The largest atomic system considered is beryllium. For Be there are no reference values of an accuracy comparable to that of $\mathrm{He}$ and $\mathrm{Li}$, therefore, the precision of the computed expectation values can be assessed merely by inspecting the convergence patterns in Table IV. The effectiveness of Dr and IT methods reminds one of that from the lithium atom
TABLE V. Hydrogen molecule, $R=1.4$ bohr. Convergence of the expectation values computed directly in comparison with those obtained by the integral transform (IT).

\begin{tabular}{|c|c|c|}
\hline$K$ & Direct & IT \\
\hline \multicolumn{3}{|c|}{$\left\langle\delta\left(\boldsymbol{r}_{i I}\right)\right\rangle$} \\
\hline 75 & 0.457670262 & 0.459447637 \\
\hline 150 & 0.458771435 & 0.459653671 \\
\hline 300 & 0.459254845 & 0.459666311 \\
\hline 600 & 0.459509138 & 0.459667951 \\
\hline 1200 & 0.459588096 & 0.459668101 \\
\hline$\infty$ & & $0.4596682(1)$ \\
\hline \multicolumn{3}{|c|}{$\left\langle\delta\left(\boldsymbol{r}_{i j}\right)\right\rangle$} \\
\hline 75 & 0.0169535 & 0.01685729 \\
\hline 150 & 0.0168143 & 0.01674822 \\
\hline 300 & 0.0167718 & 0.01674395 \\
\hline 600 & 0.0167583 & 0.01674332 \\
\hline 1200 & 0.0167616 & 0.01674339 \\
\hline$\infty$ & & $0.0167434(2)$ \\
\hline \multicolumn{3}{|c|}{$\left\langle p_{i}^{4}\right\rangle$} \\
\hline 75 & 13.18483 & 13.234252 \\
\hline 150 & 13.21449 & 13.237555 \\
\hline 300 & 13.22720 & 13.237908 \\
\hline 600 & 13.23376 & 13.237955 \\
\hline 1200 & 13.23568 & 13.237960 \\
\hline$\infty$ & & $13.237965(5)$ \\
\hline \multicolumn{3}{|c|}{$\left\langle P\left(1 / r_{i j}^{3}\right)\right\rangle$} \\
\hline 75 & 0.38592 & 0.397508 \\
\hline 150 & 0.39295 & 0.397692 \\
\hline 300 & 0.39561 & 0.397899 \\
\hline 600 & 0.39659 & 0.397956 \\
\hline 1200 & 0.39634 & 0.397944 \\
\hline$\infty$ & & $0.39795(5)$ \\
\hline
\end{tabular}

calculation-the regularization enables the stabilization of at least two additional digits. We also present our recommended values estimated by an extrapolation to the infinite basis, using data combined from Dr and IT sequences. Our result $\left\langle\delta\left(\boldsymbol{r}_{i}\right)\right\rangle=35.3695(5)$ agrees very well with 35.3700 and 35.3695 derived from the CI wave function expanded in Slater-type orbitals obtained by Bunge ${ }^{28}$ and Esquivel and Bunge, ${ }^{29}$ respectively.

Within the Dr approach, computing the necessary molecular matrix elements involves a numerical integration. For $\mathrm{H}_{2}$, therefore, we concentrated on the IT method, which is more convenient to apply, and our analysis of the convergence is based on the IT results given in Table V. The general observations are similar to that for atoms. Despite employing energetically extremely accurate wave functions (the 1200-term ECG function yields energy with an accuracy of a fraction of nanohartree), the direct computation yields only three significant digits for the Dirac deltas, four digits in case of $\left\langle p_{i}^{4}\right\rangle$, and just two for $\left\langle P\left(1 / r_{i j}^{3}\right)\right\rangle$. As we can see from the last column of Table $\mathrm{V}$, the regularization allows the next three significant figures to be recovered. Our recommended values of $\left\langle\delta\left(\boldsymbol{r}_{i I}\right)\right\rangle=0.4596682(1)$ and $\left\langle\delta\left(\boldsymbol{r}_{i j}\right)\right\rangle$ $=0.0167434(2)$ can be confronted with 0.459663 and 
TABLE VI. Convergence of $\rho$, see Eq. (32), for the hydrogen molecule at $R=1.4$ bohr.

\begin{tabular}{cc}
\hline \hline$K$ & $\rho$ \\
\hline 75 & 0.442126 \\
150 & 0.443213 \\
300 & 0.443680 \\
600 & 0.443925 \\
1200 & 0.444001 \\
$\infty$ & $0.44404(2)$ \\
\hline
\end{tabular}

0.0167740 , respectively, computed by Wolniewicz from his 279-term explicitly correlated wave function of the KołosWolniewicz type. ${ }^{30}$

For molecules, the expectation value of $p^{4}$ requires a slightly different treatment than for atoms. The molecular asymptotic formula, Eq. (31), unlike for atoms, involves an oscillatory term of the order of $p^{-7}$. The amplitude of the oscillation is determined by a new quantity, $\rho=\Sigma_{i} \rho_{i}\left(\mid \boldsymbol{R}_{A}\right.$ $-\boldsymbol{R}_{B} \mid$ ), defined in Eq. (32) and related to a nondiagonal oneelectron density matrix. In Table VI we show the convergence and the extrapolated value of $\rho$ for the hydrogen molecule at $R=1.4$. Note that for $\boldsymbol{R}_{A}=\boldsymbol{R}_{B}, \rho$ reduces to $\left\langle\delta\left(\boldsymbol{r}_{i I}\right)\right\rangle$, therefore, at small arguments $\rho$ has numerical values close to that of $\left\langle\delta\left(\boldsymbol{r}_{i I}\right)\right\rangle$. This relation can be illustrated by comparing our recommended value of $\rho=0.44404(2)$ with the density at the nucleus equal to $0.4596682(1)$. Modeling the large- $p$ momentum density using Eq. (31) enables the precision of $\left\langle p_{i}^{4}\right\rangle$ to be extended by an additional three digits in comparison with the direct evaluation (see Table V).

To our knowledge, there are no published attempts to compute the Araki-Sucher term (7) for $\mathrm{H}_{2}$. This term, together with the Bethe logarithm, is indispensable for a precise determination of the radiative energy correction of the order of $\alpha^{3}$. In Table $\mathrm{V}$ we present the convergence of $\left\langle P\left(1 / r_{i j}^{3}\right)\right\rangle$ obtained from both direct and IT computations, as well as our recommended value of 0.397 95(5).

The integral transform can also be used when the molecular wave function happens to be not very accurate or for not too short internuclear distances. Then the $A_{i}$ coefficients can be taken from the corresponding atomic systems. We have checked such an approach for the hydrogen molecule and we claim that, even for such a small internuclear distance as $R=1.4$, the atomic values of $A_{i}$ can be used, without losing much accuracy, if $t_{\Lambda} \gtrsim 100$.

\section{SUMMARY}

The main deficiency of any wave function expanded in a Gaussian basis is the lack of the cusps which results in a relatively slow convergence of expectation values of singular operators. On a few examples we have shown that this deficiency can be significantly weakened using a properly defined regularization. The new formulas for Drachmanized expectation values of singular operators enable acceleration of the convergence in the case of atoms. For molecules, how- ever, this approach requires a numerical integration and therefore becomes less practical. The integral transform of the expectation values in connection with the corrected asymptotic expansion turns out to be a convenient alternative, at least equally efficient in most cases and with a clear advantage of being easily applicable to atoms and to molecules. Both methods lead to results two to four orders of magnitude more accurate than those computed directly. Although we employed in our calculations the exponentially correlated Gaussian functions to generate benchmark results, the techniques presented in this work are, obviously, more general and can be used with any Gaussian basis. This opens the possibility of very accurate calculations of relativistic and QED effects using standard and well-established basis sets.

\section{ACKNOWLEDGMENTS}

We are indebted to B. Jeziorski and G. Łach for valuable comments on the manuscript. This work was supported by EU Grant No. HPRI-CT-2001-50034 and by the Polish State Committee for Scientific Research Grant No. SPB/COST/T09/DWM572. Support from Poznań Networking and Supercomputing Center is also gratefully acknowledged.

${ }^{1}$ W. Cencek and W. Kutzelnigg, J. Chem. Phys. 105, 5878 (1996).

${ }^{2}$ W. Cencek and J. Rychlewski, Chem. Phys. Lett. 320, 549 (2000).

${ }^{3}$ J. Komasa, J. Rychlewski, and K. Jankowski, Phys. Rev. A 65, 042507 (2002).

${ }^{4}$ J. Komasa and W. Cencek, in Computational Methods in Science and Technology (Scientific Publishers OWN, Poznań, 2003), vol. 9, p. 79.

${ }^{5}$ J. Komasa, W. Cencek, and J. Rychlewski, in Computational Methods in Science and Technology (Scientific Publishers OWN, Poznań, 1996), vol. 2, p. 87.

${ }^{6} \mathrm{~J}$. Rychlewski and J. Komasa, in Explicitly Correlated Wave Functions in Chemistry and Physics, edited by J. Rychlewski (Kluwer Academic, Dordrecht, 2003), p. 91.

${ }^{7}$ J. Komasa, J. Chem. Phys. 115, 158 (2001).

${ }^{8}$ W. Cencek, K. Szalewicz, and B. Jeziorski, Phys. Rev. Lett. 86, 5675 (2001).

${ }^{9}$ W. Cencek, in Ref. 6, p. 347.

${ }^{10}$ K. Pachucki and J. Komasa, Phys. Rev. A 68, 042507 (2003).

${ }^{11}$ K. Pachucki and J. Komasa, Phys. Rev. Lett. 92, 213001 (2004).

${ }^{12}$ K. Pachucki and J. Komasa, Chem. Phys. Lett. 389, 209 (2004).

${ }^{13}$ K. Pachucki and J. Komasa, Phys. Rev. A 70, 022513 (2004).

${ }^{14}$ W. Kutzelnigg, Theor. Chim. Acta 68, 445 (1985).

${ }^{15}$ W. Kutzelnigg and W. Klopper, J. Chem. Phys. 94, 1985 (1991).

${ }^{16}$ R. J. Drachman, J. Phys. B 14, 2733 (1981).

${ }^{17}$ K. Singer, Proc. R. Soc. London, Ser. A 258, 412 (1960).

${ }^{18}$ J. Komasa, W. Cencek, and J. Rychlewski, Phys. Rev. A 52, 4500 (1995).

${ }^{19}$ J. Komasa and J. Rychlewski, Mol. Phys. 91, 909 (1997).

${ }^{20}$ J. Komasa, J. Chem. Phys. 110, 7909 (1999).

${ }^{21}$ W. Cencek, J. Komasa, and J. Rychlewski, in Handbook on Parallel and Distributed Processing (Springer, Berlin, 2000), p. 505.

${ }^{22}$ L. Lewin, Polylogarithms and Associated Functions (Elsevier North Holland, Amsterdom, 1981).

${ }^{23}$ B. Jeziorski and K. Szalewicz, Phys. Rev. A 19, 2360 (1979).

${ }^{24}$ G. W. Drake, Nucl. Instrum. Methods Phys. Res. B 31, 7 (1988).

${ }^{25} \mathrm{G}$. W. Drake (private communication).

${ }^{26}$ K. Pachucki and J. Sapirstein, J. Phys. B 33, 455 (2000).

${ }^{27}$ Z.-C. Yan and G. W. F. Drake, Phys. Rev. A 61, 022504 (2000).

${ }^{28}$ C. F. Bunge, Int. J. Quantum Chem. 12, 343 (1977).

${ }^{29}$ R. O. Esquivel and A. V. Bunge, Int. J. Quantum Chem. 32, 295 (1987).

${ }^{30}$ L. Wolniewicz, J. Chem. Phys. 99, 1851 (1993).

${ }^{31}$ J. Komasa, Phys. Rev. A 65, 012506 (2002). 